\title{
Statistics, distillation, and ordering emergence in a two-dimensional stochastic model of particles in counterflowing streams
}

\author{
Eduardo Velasco Stock, ${ }^{1}$ Roberto da Silva, ${ }^{1}$ and H. A. Fernandes ${ }^{2}$ \\ ${ }^{1}$ Instituto de Física, Universidade Federal do Rio Grande do Sul, UFRGS, Porto Alegre, Rio Grande do Sul, 91501-970, Brasil, \\ ${ }^{2}$ Universidade Federal de Goiás - UFG, Regional Jataí, Jataí, Goiás, 75801-615, Brazil
}

(Received 24 April 2017; published 31 July 2017)

\begin{abstract}
In this paper, we propose a stochastic model which describes two species of particles moving in counterflow. The model generalizes the theoretical framework that describes the transport in random systems by taking into account two different scenarios: particles can work as mobile obstacles, whereas particles of one species move in the opposite direction to the particles of the other species, or particles of a given species work as fixed obstacles remaining in their places during the time evolution. We conduct a detailed study about the statistics concerning the crossing time of particles, as well as the effects of the lateral transitions on the time required to the system reaches a state of complete geographic separation of species. The spatial effects of jamming are also studied by looking into the deformation of the concentration of particles in the two-dimensional corridor. Finally, we observe in our study the formation of patterns of lanes which reach the steady state regardless of the initial conditions used for the evolution. A similar result is also observed in real experiments involving charged colloids motion and simulations of pedestrian dynamics based on Langevin equations, when periodic boundary conditions are considered (particles counterflow in a ring symmetry). The results obtained through Monte Carlo simulations and numerical integrations are in good agreement with each other. However, differently from previous studies, the dynamics considered in this work is not Newton-based, and therefore, even artificial situations of self-propelled objects should be studied in this first-principles modeling.
\end{abstract}

DOI: 10.1103/PhysRevE.96.012155

\section{INTRODUCTION}

In condensed matter physics, the study of the transport of particles in random environments under quenched or annealed scenarios has a huge number of applications such as the capture and decapture of electrons in the micro-, nano-, and mesodevices [1-3], the random motion of molecular motors in the cellular transport [4], the erratic motion of molecules in cromatographic columns [5], and many others. Among these problems, one in particular has called our attention: the counterflowing streams of particles, which appears in many contexts due to its importance, for instance, in the separation of chemical products [6], pedestrian dynamics [7-13], and band formation in mixtures of oppositely charged colloids $[14,15]$.

Recently, more precisely in the context of pedestrian dynamics, some authors studied the problem by considering the equation of motion for each particle as a Langevin-like equation [13] which uses the well-known and successful approach of social force developed by Helbing and Molnár in 1995 [7]. Basically, such an approach defines that the dynamics depends on deterministic and stochastic forces. They considered a Stokesian drag force that imposes velocities on the particles, an interaction force (repulsion) among particles, an interaction force of particles with the system boundaries, and a Gaussian noise term (based on the existence of an arbitrary motion in a crowd). The authors [13] showed that asymmetrically shaped walls in a corridor with pedestrian counterflow can surprisingly organize the flow of pedestrians in two only opposite directions ("keep-left" behavior). In pedestrian dynamics, the lane formation phenomenon in pedestrian counterflow was observed via numerical results in other situations. For instance, in Ref. [16], the simulations based on optimal path-choice strategy showed that the segregation was associated with the minimization of the travel time to reach the ordering state. Similarly, an extended lattice-gas model under periodic boundary conditions was also able to lead to such lane patterns [17]. Such results were experimentally corroborated by real situations as shown by Kretz et al. [18] through a pedestrian counterflow experiment in a corridor of 2-m width and 67 participants.

In addition, based on the concept of clannish random walks, Montroll and West [19] gave an alternative approach for the study of the problems of counterflowing streams of particles in a special kind of mean-field regime. By making the necessary changes and adaptations, we proposed in Ref. [12] a onedimensional stochastic dynamics based on an embryonic idea of those authors by substituting the effects of social forces and other contributions by the following assumptions: (a) from a microscopic point of view, more than one particle can occupy the same cell and (b) each particle of one species jumps to a neighbor cell with a probability that depends on the local concentration of particles of the other species.

By following the experimental results of Vissers et al. $[14,15]$ about patterns produced by oppositely charged particles under the action of electric fields, we wonder what the minimal ingredients necessary to produce lane patterns in a stochastic dynamics based on the occupation of cells might be. So, in this paper we propose a two-dimensional model which describes the motion of two species of particles $(A$ and $B)$ in opposite directions.

In our model, a particle of a given species, located on a cell, is able to move to a neighbor cell according to a transition probability that depends on the concentration of particles of the other species at the selected neighbor cell at that time. This is a general and simple way to model the possible interactions between both species.

The model was motivated by the pedestrian dynamics and the stationary cases are compared to alternative models as, for example, the one presented by the authors of Ref. [13]. 


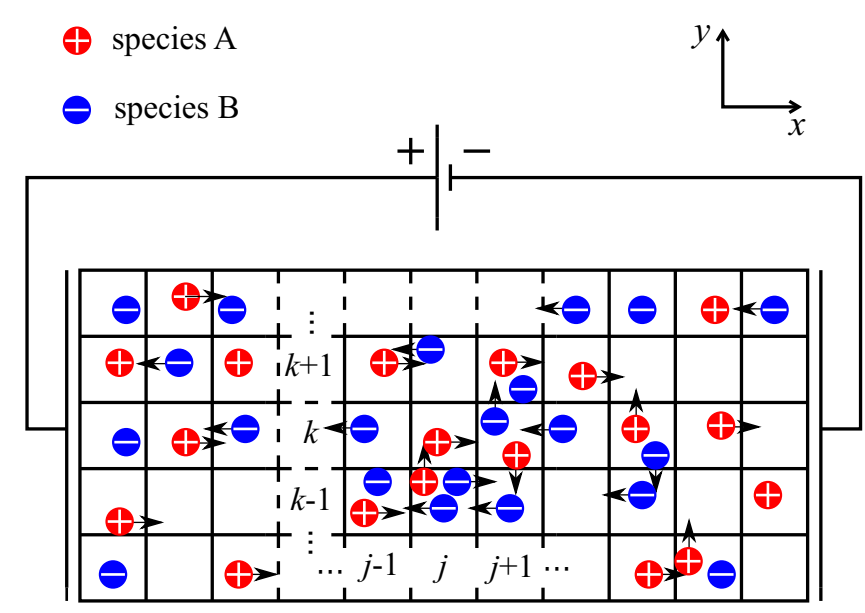

FIG. 1. Scheme of the stochastic model of two species of particles in a two-dimensional lattice where the particles can transit to the neighbor cells. In this modeling, the particles of the species $B$ are able to move or can remain in fixed cells considering the flux of the target particles of the species $A$. In a first case (annealed scenario) we are considering counterflowing streams of particles of species $A$ against particles of the species $B$. On the other hand, we can consider the particles of species $B$ working as fixed obstacles (quenched scenario). In this last case, the motion of particles $A$ is an example of the ordered transport of particles with fixed impurities spatially distributed in the environment. The analogy with a charged system and an electric field applied along the longitudinal direction is explored in this figure.

It is still important to mention that the ordering patterns in our model can be compared to similar systems based on cellular automata and lattice gas [20-23], or even with models that consider a predictive mechanism to understand the self-organization phenomena in pedestrian dynamics (see, for example, Ref. [24]) and also with experimental results [25].

The generality of our stochastic modeling offers many alternatives of scenarios as suggested in Fig. 1, and, depending on the choice of the parameters, we can apply the model for different situations.

(1) Annealed scenario: The species move against each other, for example, $A$ to the right and $B$ to the left composing two counterflowing streams of particles. The two streams interact affecting each other. In a cell, the concentration of particles of a given species changes as time evolves and therefore the transition probabilities of the another species to that cell also change according to this concentration. We can imagine, for instance, oppositely charged particles under the action of electric fields in the context of granular materials [14,15]. So we can observe jamming effects that emerge due to the resistance of the oppositely charged particles moving in opposite directions since the motion depends on their concentration per cell. Another example is the motion of the two counterflowing streams of pedestrians in a corridor. In that case, the self-propulsion of the pedestrians into a preferential direction plays the role of the electric field [12,13].

(2) Quenched scenario: The particles of the species $B$ remain in fixed positions chosen at random at the beginning of the time evolution. In this case, these particles work as obstacles for the particles of species $A$. One can imagine the particles of the species $B$ as fixed impurities in a material and the model can be thought as a traditional random (but directed) walk of particles in a general transport phenomena, exactly as happens in the electrons' transport in the interfaces of a semiconductor, in the chromatograph process of molecules, the motion in doped materials, transport in porous media and many others (see, for example, Refs. [3,5,26]).

In this paper, we describe the statistical fluctuations and the relaxation process of this stochastic model considering different initial conditions in both scenarios for a rectangular lattice that may or may not have longitudinal periodic boundary conditions depending on the studied problem. However, reflexive lateral boundary conditions are considered in all situations. We also show an approach that considers both Monte Carlo (MC) simulations and numerical integration (NI) of recurrence equations of the flows of particles along the lattice. The partial differential equations that govern the dynamics at continuum limit are also deduced.

In the annealed scenario we can separate our contribution in three main parts.

(1) Statistical characterization of spatial distribution of particles which considers one strip of each species located initially at opposite ends of the corridor.

(2) We analyze the fluctuations in the times needed to the complete separation of the two species (distillation) by considering them randomly mixed in the lattice at the beginning of the evolution.

(3) We study the emergence of ordering by longitudinal bands with periodic boundary conditions (or simply that the two species can rotate in a ring). We define an order parameter to quantify the appearance of bands considering the interaction of the counterflowing streams of the two different species.

On the other hand, in the quenched scenario, we concentrate our study in the analysis of the fluctuations on the crossing times of the target particles considering different densities of the fixed particles (obstacles). We observe crossovers at low, intermediate, and high concentrations of obstacles. For low concentration, the crossing times are normally distributed (a limited case of a negative binomial distribution), and in a transient situation, corresponding to an intermediate concentration of obstacles, they are distributed according to a power law. Finally, exponential tails appear after a high concentration of particles, making the system very stagnant.

In the next section, we explain the details of the modeling and definitions of the order parameters which describe the cellular segregation of the particles in the different studies. In Sec. III we present the results related to the situation where one of the species is not able to move, i.e., the particles of that species are fixed in the cells and work as obstacles for the oriented transport of the target particles (the particles of the other species). In Sec. IV, we present our main results when the two species are oriented in opposite directions forming counterflowing streams of particles. We finally present some summaries and our conclusions in Sec. VI.

\section{METHODS AND MODELING}

In this model, we consider a corridor with linear dimensions $L_{x}$ and $L_{y}$, the width and length dimensions, respectively, so that we have a lattice with $L_{x} L_{y}$ cells. We take into account particles of species $A(+)$ that move preferably to 
the left and particles of species $B(-)$ moving preferably to the right according to, for example, an application of a uniform field (which is supposed to be constant). We denote $\rho_{A}(j, k)=\frac{c_{A}(j, k)}{c_{A}(j, k)+c_{B}(j, k)}$ and $\rho_{B}(j, k)=\frac{c_{B}(j, k)}{c_{A}(j, k)+c_{B}(j, k)}$ as the concentration of particles $A$ and $B$, respectively, in the cell $(j, k)$ with $j=1, \ldots, L_{x}$ and $k=1, \ldots, L_{y}$. Here, $c_{A}$ and $c_{B}$ are, respectively, the total number of particles of the species $A$ and $B$ in the cell.

In the more general context (annealed system), we denote $p$ as the probability that directs the particles of both species and which depends on the applied field. So, the interaction occurs when the counterflowing particles visit the same cells. For the species $A$ this interaction is defined by (the same analysis is applied to the species $B$ ):

$$
\begin{aligned}
\operatorname{Pr}^{(A)}(j \rightarrow j+1, k \rightarrow k) & =p-\alpha \rho_{B}(j, k), \\
\operatorname{Pr}^{(A)}(j \rightarrow j, k \rightarrow k \pm 1) & =\beta_{\perp} \rho_{B}(j, k), \\
\operatorname{Pr}^{(A)}(j \rightarrow j-1, k \rightarrow k) & =\beta_{\|} \rho_{B}(j, k), \\
\operatorname{Pr}^{(A)}(j \rightarrow j, k \rightarrow k) & =1-p+(\alpha-\beta) \rho_{B}(j, k),
\end{aligned}
$$

where $\beta=2 \beta_{\perp}+\beta_{\|}$. Here $\alpha$ is related to the frontal resistance that particles offer to the reference particles as considered in Ref. [12]. The particles can move to the right or to the left with the same probability $\beta_{\perp} \rho_{B}(j, k)$. We introduce an elastic effect that promotes the return of the particle to the previous cell with probability $\beta_{\|} \rho_{B}(j, k)$.

By denoting $n_{j, k, l}$ the number of target particles in the cell $(j, k)$ at the instant $l$, the first approach leads to the recurrence equation

$$
\begin{aligned}
& n_{j, k, l+1} \\
& =n_{j, k, l}-p\left(n_{j, k, l}-n_{j-1, k, l}\right) \\
& \quad+\alpha\left[\left(1-\frac{n_{j, k, l}}{N_{j, k, l}}\right) n_{j, k, l}-\left(1-\frac{n_{j-1, k, l}}{N_{j-1, k, l}}\right) n_{j-1, k, l}\right] \\
& \quad+\beta_{\|}\left[\left(1-\frac{n_{j+1, k, l}}{N_{j+1, k, l}}\right) n_{j+1, k, l}-\left(1-\frac{n_{j, k, l}}{N_{j, k, l}}\right) n_{j, k, l}\right] \\
& \quad+\beta_{\perp}\left[\left(1-\frac{n_{j, k+1, l}}{N_{j, k+1, l}}\right) n_{j, k+1, l}+\left(1-\frac{n_{j, k-1, l}}{N_{j, k-1, l}}\right) n_{j, k-1, l}\right. \\
& \left.\quad-2\left(1-\frac{n_{j, k, l}}{N_{j, k, l}}\right) n_{j, k, l}\right],
\end{aligned}
$$

where $N_{j, k, l}=n_{j, k, l}+m_{j, k, l}$ is the total of particles in the cell $(j, k)$ at the instant $t=l \Delta, \Delta$ being the time interval among the transitions, and $m_{j, k, l}$ is the number of opposite particles in the same cell and instant.

This corresponds to the discretizations of the partial differential equation (PDE) (see [12]):

$$
\frac{\partial c_{A}}{\partial t}=-k_{1} \frac{\partial c_{A}}{\partial x}+k_{2} \frac{\partial}{\partial x}\left(\frac{c_{A} c_{B}}{c_{A}+c_{B}}\right)+k_{3} \frac{\partial^{2}}{\partial y^{2}}\left(\frac{c_{A} c_{B}}{c_{A}+c_{B}}\right),
$$

where $k_{1}=\lim _{a, \Delta \rightarrow 0} \frac{a}{\Delta} p, \quad k_{2}=\lim _{a, \Delta \rightarrow 0} \frac{a}{\Delta}\left(\alpha+\beta_{\|}\right)$, and $k_{3}=\lim _{b, \Delta \rightarrow 0} \frac{b^{2}}{\Delta} \beta_{\perp}$. The parameters $a$ and $b$ are, respectively, the dimensions of the cell. Although the numerical properties of Eq. (3) deserve attention, to compare to MC simulations, we can simply make $a=b=\Delta=1$ since each time unit corresponds to one MC step and our intention is simply to integrate the recurrence equations.

The quenched scenario is obtained simply by making $\operatorname{Pr}^{(B)}(\cdot \rightarrow \cdot)$ identically equal to zero in Eq. (1) and $\operatorname{Pr}^{(A)}(\cdot \rightarrow \cdot)$ calculated exactly as in the annealed scenario, i.e., by following Ref. [1].

\section{Order parameters: Cellular, transversal, and longitudinal segregation}

In this work, we use order parameters to measure quantitatively the patterns of separation of the particles in the lattice. For example, in models of charged colloids [14] as well as pedestrian models [13] one can observe band formation patterns. Such bands must be formed taking into account the direction of the applied field that interacts with the particles in the lattice, as well as the boundary conditions of the problem. In a general way, we can define the cellular segregation in a two-dimensional system independently of the direction at the time $t=l \Delta$ as

$$
\begin{aligned}
\Phi_{\mathrm{cell}}^{l} & =\frac{\sum_{j=1, k=1}^{L x, L y}\left|n_{j, k, l}-m_{j, k, l}\right|}{\sum_{j=1, k=1}^{L x, L y}\left(n_{j, k, l}+m_{j, k, l}\right)} \\
& =\frac{1}{N} \sum_{j=1, k=1}^{L x, L y}\left|n_{j, k, l}-m_{j, k, l}\right|,
\end{aligned}
$$

where $N \equiv N_{A}+N_{B}$ is the total number of particles in the lattice. This order parameter measures how the species are segregated by each other per cell, or simply how much the species are separated per cell. For instance, if in each cell of the lattice there is only a given species $(A$ or $B), \Phi_{\text {cell }}^{l}=1$. On the other hand, we have $\Phi_{\text {cell }}^{l}=0$ when every cell is occupied by both species in the same proportion. If we are looking for band formation (transversal or longitudinal), which are particular cases of segregation, we must define two particular segregation order parameters.

(1) The transversal segregation order parameter

$$
\begin{aligned}
\Phi_{\perp}^{l} & =\frac{\sum_{j=1}^{L x}\left|\sum_{k=1}^{L y}\left(n_{j, k, l}-m_{j, k, l}\right)\right|}{\sum_{j=1, k=1}^{L x, L y}\left(n_{j, k, l}+m_{j, k, l}\right)} \\
& =\frac{1}{N} \sum_{j=1}^{L x}\left|n_{j, l}-m_{j, l}\right|,
\end{aligned}
$$

where $n_{j, l}=\sum_{k=1}^{L y} n_{j, k, l}$ and $m_{j, l}=\sum_{k=1}^{L y} m_{j, k, l}$. This parameter measures how much the species are separated by lanes perpendicular to field, i.e., $\Phi_{\perp}^{l}=1$ if only particles of same species occupy the same transversal strip (particles in cells with a fixed $x$ coordinate). Of course, $\Phi_{\perp}^{l}=0$ if every transversal strip is occupied by both species in the same proportion.

(2) The longitudinal segregation order parameter

$$
\begin{aligned}
\Phi_{\|}^{l} & =\frac{\sum_{k=1}^{L y}\left|\sum_{j=1}^{L x}\left(n_{j, k, l}-m_{j, k, l}\right)\right|}{\sum_{j=1, k=1}^{L x, L y}\left(n_{j, k, l}+m_{j, k, l}\right)} \\
& =\frac{1}{N} \sum_{k=1}^{L y}\left|n_{k, l}-m_{k, l}\right|,
\end{aligned}
$$


where $n_{k, l}=\sum_{j=1}^{L x} n_{j, k, l}$ and $m_{k, l}=\sum_{j=1}^{L x} m_{j, k, l}$. Similarly, $\Phi_{\|}^{l}=1$ if only particles of the same species occupy the same longitudinal strip (particles in cells with a fixed $y$ coordinate). On the other hand, $\Phi_{\|}^{l}=0$ if every longitudinal strip is occupied by both species in the same proportion, indicating that this parameter measures how separated by lanes parallel to the field the species are.

Such proposed parameters are used to describe the dynamics relaxation of our model in two different contexts: (1) distillation of particles and (2) band formations when the particles are looping in a ring (the particles have periodic boundary conditions in the longitudinal direction).

\section{RESULTS I: SPECIES B AS FIXED OBSTACLES-QUENCHED SCENARIO}

First of all, we start our study performing the statistical analysis of the spatial and temporal properties of the particles. We consider the particles of the species $A$ initially disposed as a transversal strip, on the far left of the two-dimensional lattice, which means

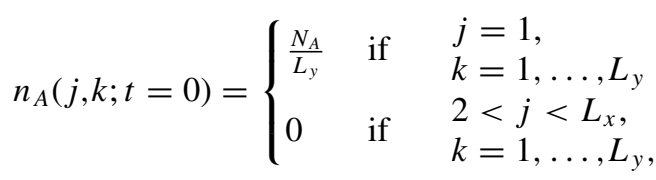

while the particles of the species $B$, at rest, are uniformly distributed in the corridor, i.e., $n_{B}(j, k ; t=0) \approx \frac{N_{B}}{L_{x} L_{y}}$. This assumption corresponds to the second scenario described in the introduction (the particles of the species $B$ are fixed obstacles to the particles of the species $A$ ). So we measured the crossing time $\tau$ (the necessary time for the particles of the species $A$ cross the corridor) to explore the effects of some parameters such as $N_{A}, N_{B}, \alpha, \beta_{\|}$, and $\beta_{\perp}$.

Some stylized facts can be observed in Fig. 2, which shows results from MC simulations for the crossing time distribution. In all situations, we use $p=0.5$ and $N_{A}=$ $10^{6}$ particles. Figure 2(a) shows the effects of different concentrations of particles of the species $B$. From the top to the bottom, we present the plots with $N_{B}=10^{3}, 10^{6}$, and $10^{8}$ (of course, $N_{B}=10^{8}$ is not a reasonable number when studying pedestrian movements), respectively. In these three situations, we use $\alpha=0.4, \beta_{\|}=0.1$, and $\beta_{\perp}=0$. We measure the kurtosis of distribution for each case, calculated as $\left\langle\left(\frac{\tau-\langle\tau\rangle}{\delta}\right)^{4}\right\rangle$, which measures the weight of the tail of the crossing time distribution. Here $\left\langle\tau^{k}\right\rangle=\sum_{\tau} \tau^{k} f(\tau) / \sum f(\tau)$, where $f(\tau)$ is the frequency count of crossing times. Here $\delta=$ $\sqrt{\left\langle\tau^{2}\right\rangle-\langle\tau\rangle^{2}}$ is the standard deviation of the crossing time distribution.

Figure 2(b) shows the effects when different values of $\alpha$ are considered. Here we keep $N_{B}=10^{8}$ and $\beta_{\|}=\beta_{\perp}=0$. We can observe that for $\alpha=0.4$ the deviation of a Gaussian fit (continuous curve) is easily observed. Finally, the Fig. 2(c) shows two different effects: different values of $\beta_{\|}$for the same $\beta_{\perp}$ and different values of $\beta_{\perp}$ for the same $\beta_{\|}$. As can be seen, for fixed obstacles, we cannot observe a strong influence of $\beta_{\perp}$ on the crossing times, i.e., the lateral motion is not important since the particles that move laterally (changing lanes) end up encountering other obstacles whereas they are uniformly
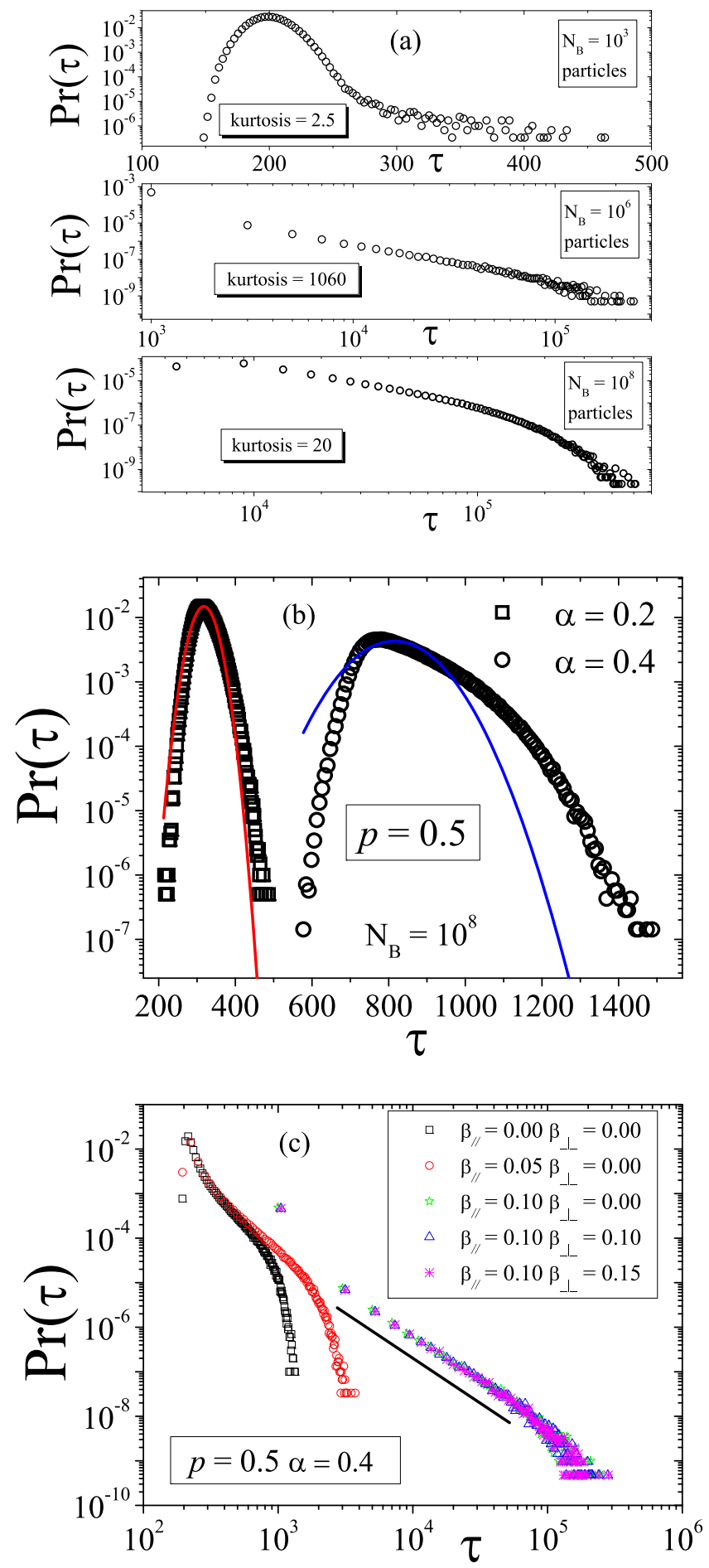

FIG. 2. MC simulations for the crossing time distribution. In these plots we use $p=0.5, L_{x}=100$, and $L_{y}=10$. Plot (a) shows the effects of the concentration of particles of the species $B$. From the top to the bottom, we use $N_{B}=10^{3}, 10^{6}$, and $10^{8}$ particles, respectively. In these three situations, we keep $\alpha=0.4, \beta_{\|}=0.1$, and $\beta_{\perp}=0$. Plot (b) shows the effects of different values of $\alpha$. Here we also keep $N_{B}=10^{8}$ and $\beta_{\|}=\beta_{\perp}=0$. Plot (c) shows two effects obtained by considering different values of $\beta_{\|}$for the same $\beta_{\perp}$ and different values of $\beta_{\perp}$ for the same $\beta_{\|}$.

distributed in the lattice. Different results are obtained in the next section, when one takes into account a lattice without 


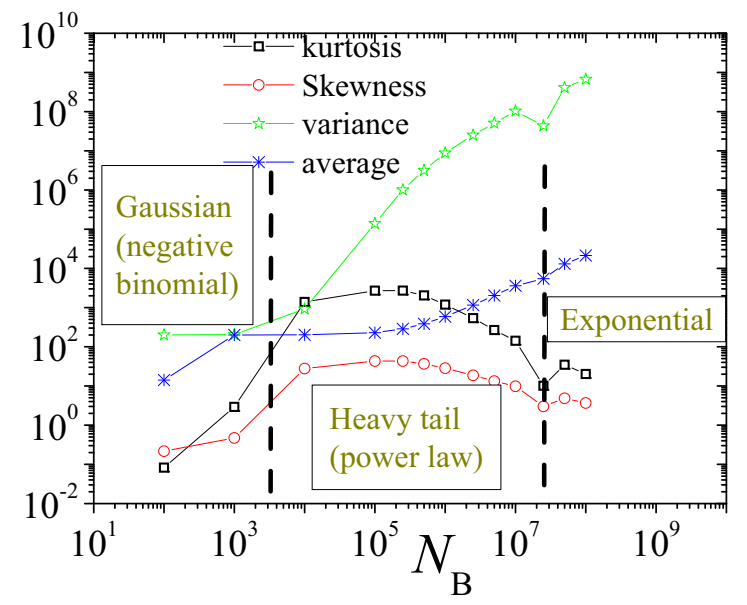

FIG. 3. Average, variance, skewness, and kurtosis of the crossing time distribution as function of $N_{B}$. By looking at kurtosis (weight tail) of the distribution, we can observe a crossover: Gaussian behavior $\rightarrow$ power-law behavior $\rightarrow$ exponential behavior. Here we use $L_{x}=$ 100 and $L_{y}=10$.

fixed obstacles, i.e., when both species of particles are able to move in opposite directions.

Let us return to Fig. 2(a) to look into some important details. There is an interesting crossover for the crossing time distribution as a function of $N_{B}$. We have a small kurtosis for the top plot $(\sim 2.5)$, a large kurtosis for the middle plot $(\sim 1060)$, and an intermediate kurtosis for the bottom plot $(\sim 20)$. This transition has a simple explanation: for low values of $N_{B}$, one has, in the first approximation, a negative binomial distribution (a Gaussian distribution at the limit) with a scattering in the tail since there is no influence of obstacles and the particles have to perform $L_{x}$ movements with $L_{x} / p$ trials until completing the path. When one has an intermediate value of $N_{B}$, an interesting phenomenon arises: although there is a more probable crossing time, torn particles (particles that tear apart from the main group of their species) are blocked by obstacles since our model is based on relative density, and therefore, clusters composed by larger numbers of particles of the same species have more advantage against the particles of the other species. These torn particles correspond to Levy flights since they have huge crossing times when compared to the other ones. Finally, when $N_{B}$ is very large, all particles have a hard task to cross the corridor, and differently from the intermediate situation, there are not very different crossing times leading to an exponential behavior.

These crossovers among Gaussian, power law, and exponential behaviors are shown in Fig. 3 through the analysis of the average, variance, kurtosis, and skewness as function of $N_{B}$. As can be seen, while the average and variance have a monotonic behavior, the kurtosis and skewness have at first an increase and then they decrease, corroborating the crossover.

\section{RESULTS II: SPECIES B IN MOTION-ANNEALED SCENARIO}

Now we consider that the particles of the species $B$ are also able to move. First, we analyze the fluctuations of spatial particle distribution by considering two opposite streams of


FIG. 4. (a) Density of particles $A$ ( $B$ is symmetric since the initial conditions are similar) in different times of evolution. (b) Time evolution of the fluctuations of the density of particles: (i) average (log-log scale), (ii) standard deviation (log-log scale), (iii) skewness, and (iv) kurtosis. In both plots we use $L_{x}=100, L_{y}=10$, and points correspond to $\mathrm{MC}$ simulations and lines to he results obtained from NI. The inset plots in the two upper figures correspond to the current plot in linear scale.
- species A
- species B
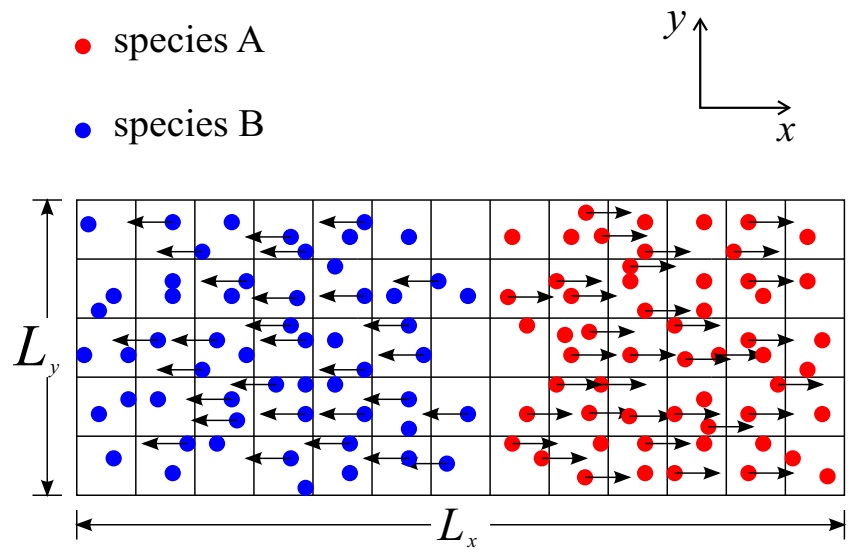

FIG. 5. Illustration of a complete distillation between the species $A$ and $B$. This means that there is a vertical line separating the two species in the corridor. We adopted $L_{x}=100$ and $L_{y}=40$ for these simulations. 
particles starting as two strips at the ends of the corridor (next subsection). Right after, in the second subsection, we consider the two different species randomly distributed and mixed in the corridor and we analyze the fluctuations on the distillation time, i.e., the needed time to separate the two species.

\section{A. Properties of the spatial particle distribution}

As we already explored the effects of crossing times of particles of the species $A$ when particles of the species $B$ work as fixed obstacles, now we would like to study some effects when the particles of the species $B$ are also able to move in the lattice. For this purpose, we analyze the properties of a scenario where two streams of different species of particles interact with each other when moving in opposite directions. So, we consider the condition previously set up to the particles of the species $A$ as valid for both species. The condition for


FIG. 6. Color map of the distillation time for different values of $\beta_{\|}$and $\beta_{\perp}$ considering different concentrations of particles for two different values of $\alpha$. In this study, we adopted $L_{x}=100$ and $L_{y}=40$.

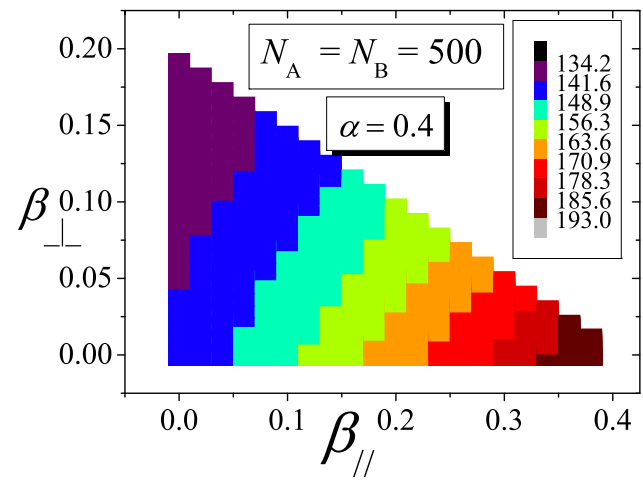

Here we are interested in the study of the scenario when the two species meet each other and interact by following the dynamics previously defined in this work. After starting the evolution of the two strips, we follow the time evolution of the shape of the density of particles for several times as can be seen in Fig. 4(a) as well as of the time evolution of the parameters which describe such density, i.e., its fluctuations [Fig. 4(b)].

We can observe in Fig. 4(a) that the density of particles $c(x, t)$ (at cell $j=x / a$ and time $t=l \Delta$ ) is deformed during the interaction of the species from $t=85$. Here it is important

to mention that a marginalization over $y$ dimension was conveniently performed according to our objectives [i.e., $\left.c(x, t)=\sum_{y} c(x, y, t)\right]$. Figure $4(\mathrm{~b})$ shows that this deformation is captured by valleys in the skewness [plot (iii)]: $\left[\sum_{x}\left(\frac{x-\langle x(t)\rangle}{\sigma(t)}\right)^{3} c(x, t)\right] / \sum_{x} c(x, t)$ and peaks in the kurtosis [plot (iv)]: [ $\left.\sum_{x}\left(\frac{x-\langle x(t)\rangle}{\sigma(t)}\right)^{4} c(x, t)\right] / \sum_{x} c(x, t)$, with $\langle x(t)\rangle=$ $\left[\sum_{x} x c(x, t)\right] / \sum_{x} c(x, t)$ and $\sigma(t)=\left[\left\langle x^{2}(t)\right\rangle-\langle x(t)\rangle^{2}\right]^{1 / 2}$ that increases in absolute value during the evolution, leaving the Gaussian behavior [represented by the distribution for $t=$ 75 as shown in Fig. 4(a)] to become a heavy tail distribution. We also can observe that such anomalous behavior is also captured by the average position $\langle x(t)\rangle$, in the plot (i) as well as by the standard deviation of the position $\sigma(t)$ of the particles according to plot (ii). In both plots we use $\alpha=0.49$, $\beta_{\|}=0.245$, and $\beta_{\perp}=0.1225$. In Figs. 4(a) and 4(b) we also present the comparison between the results obtained through MC simulations and PDE solutions. As shown, they are in good agreement to each other.

\section{B. Distillation time}

As the two species of particles follow opposite directions, an interesting situation can be observed when we mix them in the corridor at the initial stage of the evolution and analyze the needed time to a complete separation of the two species (distillation). In Fig. 5 we show a typical situation in which the species are completely distilled.

The needed time for the system to achieve the complete separation of the species is computed through numerical MC simulations for all possible pairs $\left(\beta_{\|}, \beta_{\perp}\right)$ for two different values of $\alpha$, one low (0.1) and another high (0.4). This procedure was repeated considering three different number of particles $N=N_{A}=N_{B}=100,500$, and 1000. Here, the main idea is to check if $\beta_{\perp}$ can affect the distillation time. As stated in the previous section, this parameter has no influence on the crossing times when one of the species works as a fixed obstacle. Figure 6 shows that for $\alpha=0.1$ the effects of $\beta_{\perp}$ on the distillation times are not perceptible. However, $\alpha=0.4$ leads to a reduction of the distillation time.

This reduction is more relevant when $\beta_{\|}$is higher, i.e., the movements to the sides are important when the combination of the resistance factor $(\alpha)$ and the frontal collision effects $\left(\beta_{\|}\right)$lead to a real stagnation in the movement ability. Here we monitor the order parameters $\Phi_{\text {cell }}(t)$ and $\Phi_{\perp}(t)$ as a function of time during the distillation previously described in Fig. 6. We hope that when $t \rightarrow \infty$, both $\Phi_{\text {cell }}$ and $\Phi_{\perp} \rightarrow 1$ since the system is completely distilled.

In Fig. 7 we show that both parameters converge to 1 (indicating that the system is completely distilled), and the segregation is greater as $\beta_{\perp}$ increases. A simple analysis of the finite-size scaling effects of the average time of the distillation time is shown in the inset of this figure. This plot is obtained for the worst case $\left(\beta_{\perp}=0\right.$ and the maximal allowed $\beta_{\|}$, i.e., $\left.\beta_{\|}=\alpha\right)$ as a function of the inverse number of particles in the system $N^{-1}=N_{A}^{-1}=N_{B}^{-1}$, and is presented in log-log scale for the two studied values of $\alpha$.

Whereas we already explored the properties of the distillation time and the dependence of the different factors that change this quantity, we study, in the following section, the

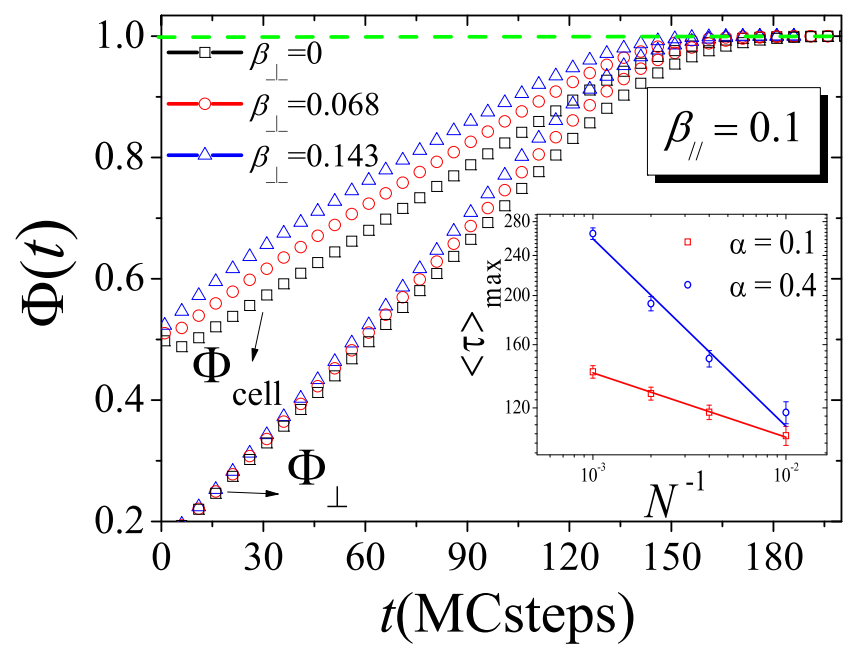

FIG. 7. Time evolution of the order parameters $\Phi_{\text {cell }}$ and $\Phi_{\perp}$ for $\beta_{\|}$fixed and for different values of $\beta_{\perp}$. This plot shows the results for $L_{x}=100$ and $L_{y}=40$. However, similar results were obtained for other lattice sizes. We can observe that both parameters converge to 1 (indicating that the system is completely distilled), and the segregation is greater as $\beta_{\perp}$ increases. The inset plot shows as the distillation times scale with the number of particles.

band formation that appears when the particles move in a ring which is obtained by considering periodic boundary conditions in the longitudinal direction.

\section{RESULTS III: PERIODIC BOUNDARY CONDITIONS-MOTION IN A RING}

Now we consider the two species of particles moving in a ring, in the opposite direction, i.e., one species of particles is moving counterclockwise and the another one is moving clockwise. Alternatively, this situation can be thought of as if particles could enter the corridor according to some rate if they are of the same species as those which are reaching the end of the corridor (ring topology). The idea is represented in Fig. 8.

First of all, we perform MC simulations considering that the species have the same number of particles $\left(N_{A}=N_{B}=\right.$

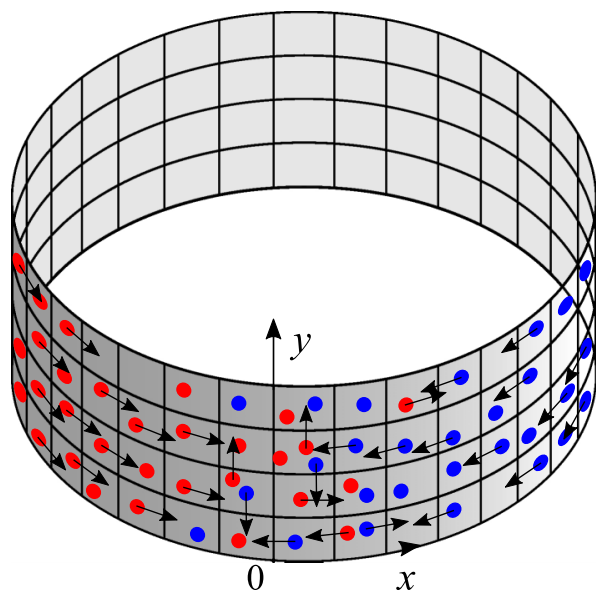

FIG. 8. Counterflow of particles of different species moving in a ring. 

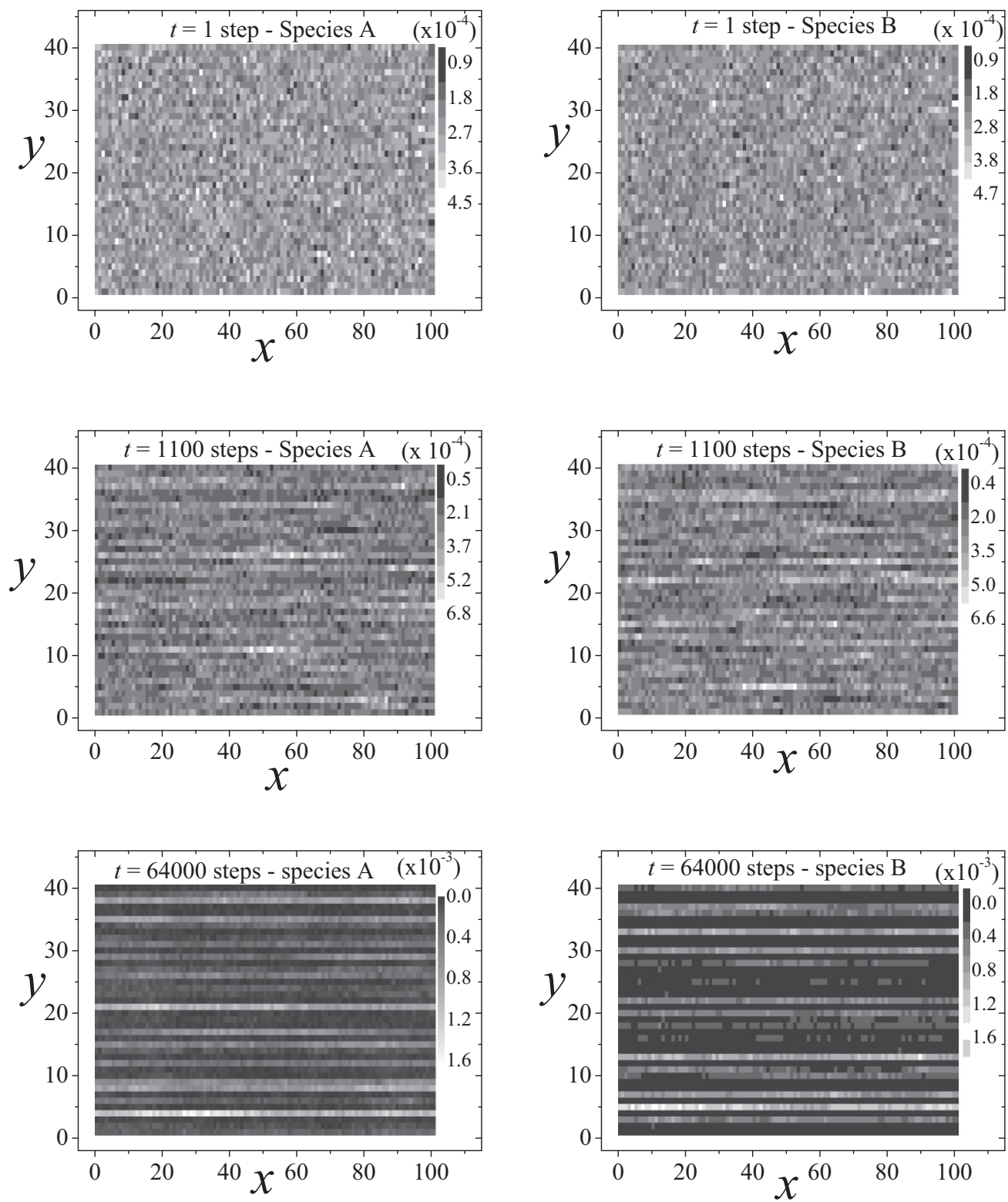

FIG. 9. Frames of the density of particles of the species $A$ (left side) and $B$ (right side) for different instants of time when they are moving in the opposite direction. The particles are uniformly distributed at the beginning of the evolution and follow the ring topology. Band formations can be observed at the end of the evolution.

$10^{5}$ ) and are uniformly distributed in the corridor. Thus, we monitor the concentrations of particles in the corridor as the time evolves, for a typical set of parameters $\alpha=0.45$ and $\beta_{\|}=\beta_{\perp}=0.15$, to observe the process of relaxation toward the formation of bands. We analyze the frames for three different MC steps: $t=1,1100$, and 64000 (see Fig. 9). Band formations can be observed after a long time corroborating the ordering of the species, which move in the same direction in the stationary stage of the evolution. Here, as well as in all results explored in this section, we fixed $L_{x}=100$ and $L_{y}=40$.

Such patterns can be better understood if we analyze the order parameters $\Phi_{\|}(t), \Phi_{\text {cell }}(t)$, and $\Phi_{\perp}(t)$ as a function of time. We can observe in Fig. 10 that $\Phi_{\|}(t)$ goes asymptotically to 1 , which corroborates the longitudinal bands observed in
Fig. 9. Likewise, we can see that $\Phi_{\text {cell }}(t)$ converges to 1 since a segregation by bands (longitudinal or vertical) implies in a segregation by cells. However, $\Phi_{\perp}(t)$ possesses lower values showing that the segregation by vertical bands (perpendicular to the orientation field of particles) is not expected.

Here one can make an analogy with some real physical systems. Vissers et al. [14] observed lane formation in driven mixtures of oppositely charged colloids when considering periodic boundary conditions and their results corroborate our theoretical findings in a purely stochastic model (see Fig. 1 in Ref. [14]). In another publication, Vissers etal. [15] showed that vertical bands appear when one applies an ac field. In an upcoming paper we will explore the effects due to an ac field in a stochastic model to reproduce such experimental results. 


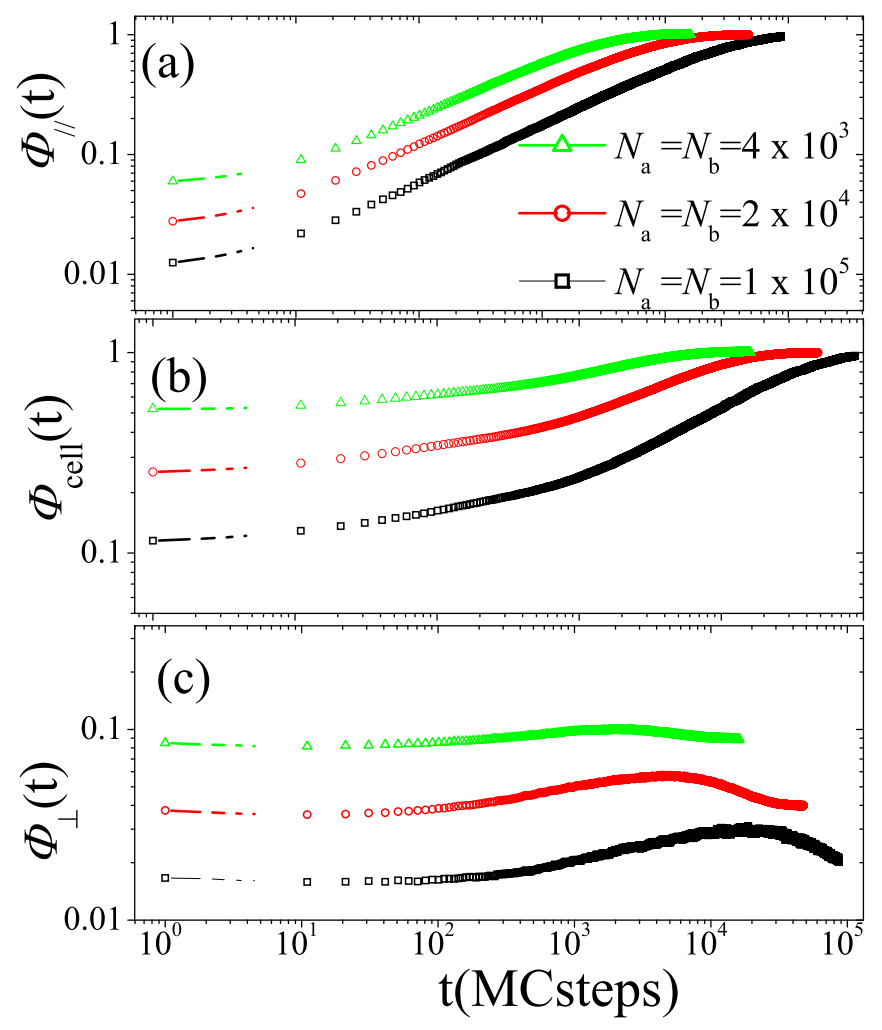

FIG. 10. Relaxation of the order parameters. We can see that $\Phi_{\|}$ and $\Phi_{\text {cell }}$ go to 1 when $t \rightarrow \infty$, corroborating the ordering of the species in longitudinal bands. However, the segregation by bands in the perpendicular direction to the field is not observed.

In a recent work, Oliveira et al. [13] proposed a model of pedestrian behavior and obtained a two-lane ordered state emerging with asymmetrically shaped walls. They were are able to organize the flow of pedestrians moving in opposite directions by solving Langevin-like equations with Stokesian drag force. Inspired by this work, we propose a computational experiment by using our model. However, differently from that study, we focus our attention to the initial conditions of the particles. We start with the particles of the species $A$ initially concentrated in a vertical stripe asymmetrically distributed along $y$ :

$n_{A}(j, k ; t=0)=\left\{\begin{array}{lll}\frac{2 N_{A}}{L_{y}}\left(L_{y}-k\right) & \text { if } & j=1, k=1, \ldots, L_{y} \\ 0 & & \\ 0 & \text { if } \quad \begin{array}{l}2 \\ k\end{array}=1, \ldots, L_{y},\end{array}\right.$

and the particles of the species $B$ are uniformly distributed on the corridor $n_{B}(j, k ; t=0)=N_{B} /\left(L_{x} L_{y}\right)$, with $j=1, \ldots, L_{x}$ and $k=1, \ldots, L_{y}$. The highlight here is the possibility of obtaining a stationary state with a two-lane ordered state if the initial condition defined above is capable of inducing a similar stationary state in $B$. We solve the problem using MC simulations and numerical integration of Eq. (2) to compare the stages of evolution. In Fig. 11, we show the frames for six different instants of time. The blue (red) color means higher (lower) concentrations of a given species.
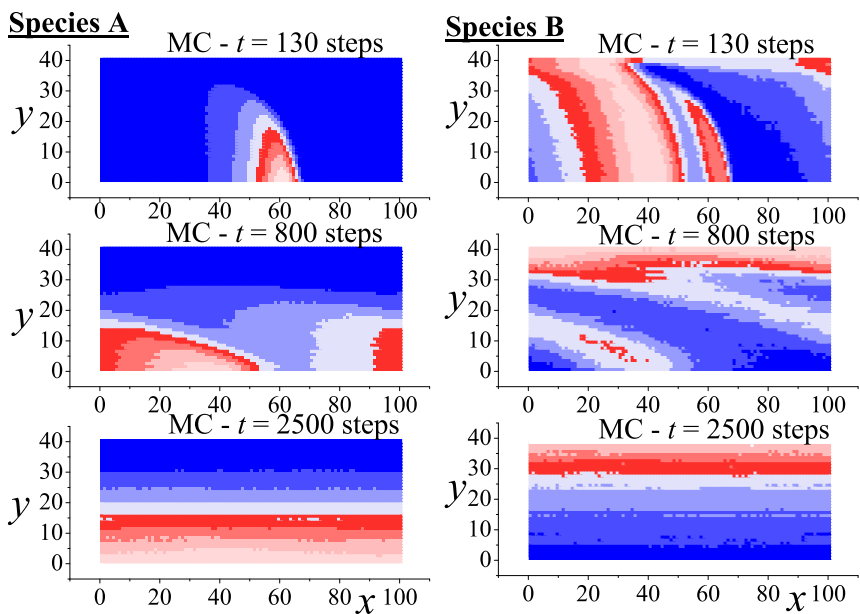

FIG. 11. Frames of some instants of time using MC simulations. We can observe stationary states with two well-distinct emergent ordered lanes. The blue (red) color means higher (lower) concentrations of the species $A$ on the left plot and $B$ on the right plot. An asymmetry is initially considered for species $A$ according to Eq. (7) while the species $B$ is uniformly distributed.

We can observe that, even with an asymmetric initial condition, the system has the tendency to reach a stationary order formed by two ordered lanes differently from what happens when one considers the initial conditions where the particles of both species are uniformly distributed. Figure 12 shows the frames for the same instants of time when one takes into consideration the numerical integration of Eq. (2). Both methods lead to stationary states with two emergent ordered lanes. Here it is important to observe that the numerical integration of equations correspond to the mean-field regime of MC simulations, and although we have a similar evolution and the same stationary state, the relaxations are not synchronized. This is exactly what should occur in statistical mechanics
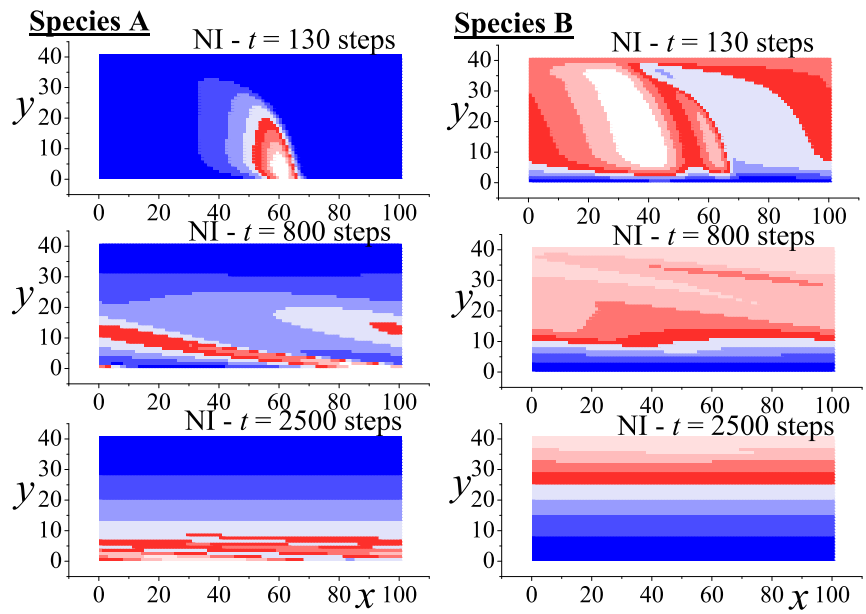

FIG. 12. Frames obtained via numerical integration of recurrence relations for six different instants of time (as in Fig. 11). The blue (red) color means higher (lower) concentrations of the species $A$ on the left plot and $B$ on the right plot. An asymmetry is initially considered for species $A$ according to Eq. (7) while the species $B$ is uniformly distributed. 

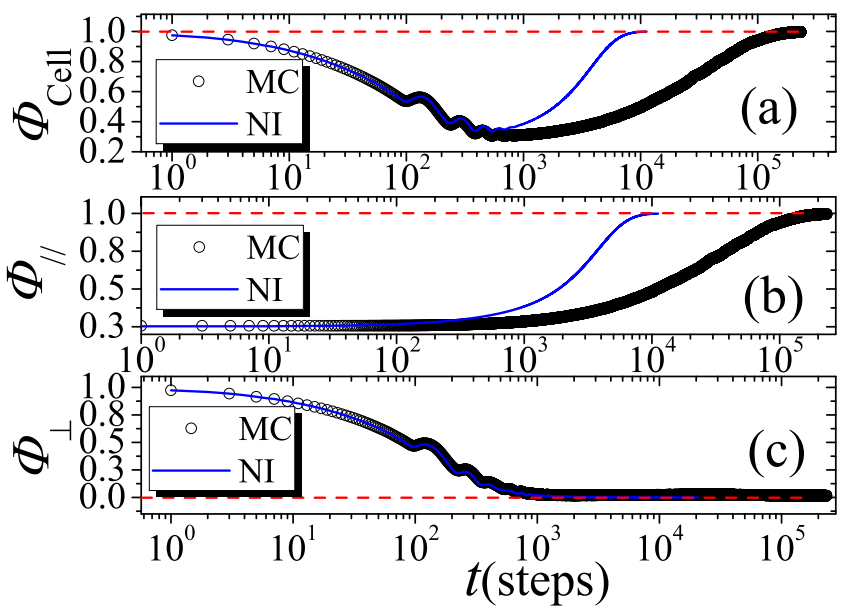

FIG. 13. Time evolution of the order parameters considering that the species $A$ is asymmetrically distributed in an initial vertical stripe according to Eq. (7) and the species $B$ is uniformly distributed in the lattice. Both methods (MC simulations and NI) lead to stationary states as previously shown in Figs. 11 and 12 composed by two emergent ordered bands which are quantitatively corroborated by the order parameters $\Phi_{\text {cell }} \rightarrow 1$ (a) and $\Phi_{\|} \rightarrow 1$ (b), when $t \rightarrow \infty$. We also show the behavior of $\Phi_{\perp}$ as a function of time. In this case $\Phi_{\perp} \rightarrow$ 0 since the particles do not present order by bands perpendicular to the field.

when, for example, we solve the Ising model by performing MC simulations and via the mean-field approach. In these cases, the critical exponents associated to possible phase transitions are different. However, we expect that $\Phi_{\|} \rightarrow 1$ in both cases, though with different speeds since Figs. 11 and 12 present different time scales of relaxation dynamics.

Figure 13 shows the time evolution of the three order parameters of the model. These evolutions are obtained when carrying out the study corresponding to the Fig. 11 (MC simulations) and Fig. 12 (NI).

We can observe in Fig. 13 that $\Phi_{\text {cell }} \rightarrow 1$ and $\Phi_{\|} \rightarrow 1$ (using MC simulations and also NI) show the stationary case formed by two main bands corresponding to the two species. The observed difference in the plots are acceptable since we do not expect the same speed in the two solutions but instead we expect the reproduction of the same steady states. However, the initial part is exactly the same for all order parameters and only the conduction to the steady state is slower for the MC simulations. Such a problem shows that an initial order of species $A$ is broken after a transient regime reaches an ordered dynamics.

\section{SUMMARY AND CONCLUSION}

We consider a two-dimensional system composed by particles of two species, $A$ and $B$, which are able to move or not depending on the scenario. If one of the species remains still, it acts as a fixed obstacle to the other species, which is moving. In this case, we use MC simulations and concentrate our analysis in the statistics about crossing times (which means the needed time for one particle to cross the lattice) for different concentrations of obstacles. However, if both species are moving, then they move in opposite directions. In this case, we look into the properties of distillation times of the particles arranged randomly in the lattice at the beginning of the evolution, as well as the formation of ordering patterns considering periodic boundary conditions of particles. Both studies are performed by using MC simulations and numerical integration of recurrence relations. In the first study, we show that transitions among the Gaussian, power law, and exponential behavior are observed for the crossing times of the particles, which is supported by high kurtosis values. We also show that the crossing times are not affected by lateral transitions. In the second study, when both species can move, we observe that enlarging the lateral transitions decreases the distillation times and the particles are more easily separated.

Finally, we observe characteristic ordering patterns when we take into consideration periodic boundary conditions, exactly as happens in charged colloids motions [14,15] and pedestrian dynamics [13,20-25]. The lane formation can be observed for the proposed order parameters when considering different initial conditions of particles $A$ and $B$, showing that steady state seems to be robust in relation to initial conditions. Still, in this context of self-propelled agents, it is important to mention the results obtained by Dickman [27], who observed a similar phenomenon related to spontaneous spatial ordering in a driven lattice-gas, but quite contrary to the usual relation between bias and current, whereas in that work the drive provoked jamming, and thereby, a sharp reduction in the current. Studies like this deserve more attention and will be taken into consideration in our future works. Still, we intend to explore exclusion effects in other contributions, for example, by introducing in our model cells with a limited maximum number of particles, and analyze these effects into ordering formation.

\section{ACKNOWLEDGMENTS}

This research work was, in part, supported financially by National Council for Scientific and Technological Development (CNPq) by Grants No. 458498/2014-9 and No. 310017/2015-7.
[1] S. Machlup, J. Appl. Phys. 25, 341 (1954).

[2] M. J. Kirton and M. J. Uren, Adv. Phys. 38, 367 (1989).

[3] R. da Silva, L. C. Lamb, and G. I. Wirth, Philos. Trans. R. Soc. A 369, 307 (2010); R. da Silva, L. Brusamarello, and G. Wirth, Physica A 389, 2687 (2010); R. da Silva and G. I. Wirth, J. Stat. Mech. (2010) P04025; R. da Silva, G. I. Wirth, and L. Brusamarello, Int. J. Mod. Phys. B 24, 5885 (2010); J. Stat. Mech. (2008) P10015.
[4] S. P. Gross, Phys. Biol. 1, R1 (2004); L. W. Rossi, P. K. Radtke, and C. Goldman, Physica A 401, 319 (2014); C. Goldman, J. Stat. Phys. 140, 1167 (2010).

[5] J. C. Giddings and H. Eyring, J. Phys. Chem. 59, 416 (1955); R. da Silva, L. C. Lamb, E. C. Lima, and J. Dupont, Physica A 391, 1 (2012).

[6] Y. Li, X. Li, Y. Wang, Y. Yin Chen, J. Ji, Y. Yu, and Z. Xu, Ind. Eng. Chem. Res. 53, 4821 (2014). 
[7] D. Helbing and P. Molnar, Phys. Rev. E 51, 4282 (1995).

[8] D. Helbing, I. J. Farkas, and T. Vicsek, Nature (London) 407, 487 (2000).

[9] A. Nakayama, K. Hasebe, and Y. Sugiyama, Comput. Phys. Commun. 177, 162 (2007).

[10] P. Gawronski and K. Kułakowski, Comput. Phys. Commun. 182 , 1924 (2011).

[11] Y.-C. Peng and C.-I. Chou, Comput. Phys. Commun. 182, 209 (2011).

[12] R. da Silva, A. Hentz, and A. Alves, Physica A 437, 139 (2015).

[13] C. L. N. Oliveira, A. P. Vieira, D. Helbing, J. S. Andrade, Jr., and H. J. Herrmann, Phys. Rev. X 6, 011003 (2016).

[14] T. Vissers, A. Wysocki, M. Rex, H. Lowen, C. P. Royall, A. Imhof, and A. van Blaaderen, Soft Matter 7, 2352 (2011).

[15] T. Vissers, A. van Blaaderen, and A. Imhof, Phys. Rev. Lett. 106, 228303 (2011).

[16] T. Xiong, P. Zhang, S. C. Wong, C. W. Shu, and M. P. Zhang, Chin. Phys. Lett. 28, 108901 (2011).

[17] X. Li, X. Y. Duan, and L.Y. Dong, Chin. Phys. B 21, 108901 (2012).
[18] T. Kretz, A. Grünebohm, M. Kaufman, F. Mazur, and M. Schreckenberg, J. Stat. Mech. (2006) P10001.

[19] E. W. Montroll and B. West, On Enriched Collection of Stochastic Process: In Fluctuation Phenomena, edited by E. W. Montroll and J. Lebowitz (Elsevier, Amsterdam, 1979).

[20] Y. Tajima, K. Takimoto, and T. Nagatani, Physica A 313, 709 (2002).

[21] W. G. Weng, S. F. Shen, H. Y. Yuan, and W. C. Fan, Physica A 375, 668 (2007).

[22] S. Nowak and A. Schadschneider, Phys. Rev. E 85, 066128 (2012).

[23] S. Xue, B. Jia, and R. Jiang, J. Stat. Mech. (2016) 113204.

[24] Z. Wang, J. Ma, H. Zhao, Y. Qin, and L. Jia, Phys. A: Math. Theor. 45, 305004 (2012).

[25] M. Isobe, T. Adachi, and T. Nagatani, Physica A 336, 638 (2004).

[26] Advances in Porous Media, Vol. 3 edited by M. Y. Corapcioglu, (Elsevier, Amsterdam, 1996).

[27] R. Dickman, Phys. Rev. E 64, 016124 (2001). 